JOURNAL OF

SYMPLECTIC GEOMETRY

Volume 11, Number 1, 25-36, 2013

\title{
A NEW BOUND ON THE SIZE OF SYMPLECTIC 4-MANIFOLDS WITH PRESCRIBED FUNDAMENTAL GROUP
}

\author{
JONATHAN T. YAZINSKI
}

\begin{abstract}
Given any finitely presented group with $g$ generators and $r$ relations, we produce a symplectic 4-manifold of Euler characteristic $10+4(g+r)$ and signature -2 . This is an improvement on the result in [BK2], and our construction utilizes a construction in [FPS].
\end{abstract}

\section{Introduction}

It is well known that any finitely presented group is the fundamental group of a closed smooth 4-manifold. The less obvious result that any finitely presented group is the fundamental group of a closed symplectic 4-manifold is shown in $[\mathbf{G}]$. In $[\mathbf{B K 2}]$, Baldridge and Kirk improve upon Gompf's construction, producing a smaller closed symplectic 4-manifold subject to the constraint of having a specified fundamental group, where the size of a manifold is measured by the magnitude of its signature and Euler characteristic. In this paper, we improve upon this result even further. Our main theorem is

Theorem 1.1. Let $G$ be a group with a presentation with $g$ generators and $r$ relations. Then there is a symplectic 4-manifold $X$ with $\pi_{1}(X)=G$ and $\chi(X)=10+4(g+r)$ and $\sigma(X)=-2$.

The construction here follows that in [BK2] closely. The main difference is that we utilize a manifold constructed in $[\mathbf{F P S}]$ in lieu of a larger one. The tricky point to consider is that the homology $S^{2} \times S^{2}$ manifolds of [FPS] are not known to be simply connected; however, since killing one generator of their fundamental group will make the group trivial, we can still carefully utilize these manifolds to obtain the desired relation in the construction below.

Remark 1.2. For any finitely presented group $G$, the manifold $X$ of Theorem 1.1 contains a symplectic torus such that the fundamental group of its 
complement is $G$. By performing a family of torus surgeries, one can obtain a countably infinite family of manifolds with the same homology and fundamental group $G$, which are pairwise non-diffeomorphic, distinguished by their Seiberg-Witten invariants. (See [MMS] and Section 2.8 of $[\mathbf{F S}]$, for example.)

\section{The construction}

We will not carefully reiterate all of the work done in $[\mathbf{B K 2}]$, as the construction here is similar. We will only outline the construction and highlight what is different.

First, let $G$ be a finitely presented group with generators $x_{1}, \ldots, x_{g}$ and relations $w_{1}, \ldots, w_{r}$. Hence

$$
G=\left\langle x_{1}, \ldots, x_{g} \mid w_{1}, \ldots, w_{r}\right\rangle .
$$

Recall that the construction in [BK2] involves considering $N \times S^{1}$, where $N$ is a surface bundle over $S^{1}$ with fiber a surface of genus $g n$, where

$$
n=1+\sum_{i=1}^{r} n_{i}
$$

and $n_{i}$ is the length of the relation $w_{i}$. The manifold $N \times S^{1}$ admits a symplectic form [Th]. Let $F$ be a surface of genus $g n$, which we think of in the following way: Let $R$ be the $\frac{2 \pi}{n g}$ rotation of $S^{2} \subseteq \mathbb{R}^{3}$ through the axis passing through the points $(0,0, \pm 1)$ in $S^{2}$. Let $D$ be a disc centered at $(1,0,0)$ whose translates under the action of $\langle R\rangle \subseteq S O(3)$ are disjoint. Then if we connect sum $S^{2}$ with a torus at each translate of $D$, we obtain a surface $F$ with a natural action of $\mathbb{Z} /(n g)$ on this surface. The surface bundle $N$ above is the bundle determined by the order $n$ monodromy $R^{g}$.

The monodromy $R^{g}$ has a fixed point (in fact, two fixed points), which we call $p$. We can write $N$ as $(F \times I) / \sim$, where $(x, 0) \sim\left(R^{g}(x), 1\right)$, and so we let $\tau$ denote the loop in $N$ that is the image of $\{p\} \times I$ under the projection to $N$ given by $\sim$. This is a loop since $R^{g}(p)=p$. We let $t \in \pi_{1}\left(N \times S^{1}\right)$ denote the element obtained by including $\tau \subseteq N=N \times\{1\}$ into $N \times S^{1}$. Also, we let $s$ denote a generator of $\{1\} \times \mathbb{Z} \subseteq \pi_{1}(N) \times \pi_{1}\left(S^{1}\right)=\pi_{1}\left(N \times S^{1}\right)$.

Now we form a new presentation of $G$, by adding $g$ generators, changing the relations, and adding $g$ more relations. We add the $g$ generators $y_{1}, \ldots, y_{g}$, and we add the $g$ relations $x_{1} y_{1}, \ldots, x_{g} y_{g}$, and for each relation $w_{i}$, we obtain a corresponding relation $\tilde{w}_{i}$. The relation $\tilde{w}_{i}$ is obtained from the relation $w_{i}$ by replacing occurrences of $x_{k}^{-1}$ with $y_{k}$. Hence, each relation is expressed as a product of positive powers of the generators. The new presentation for $G$ is

$$
G=\left\langle x_{1}, \ldots, x_{g}, y_{1}, \ldots, y_{g} \mid \tilde{w}_{1}, \ldots \tilde{w}_{r}, x_{1} y_{1}, \ldots, x_{g} y_{g}\right\rangle
$$




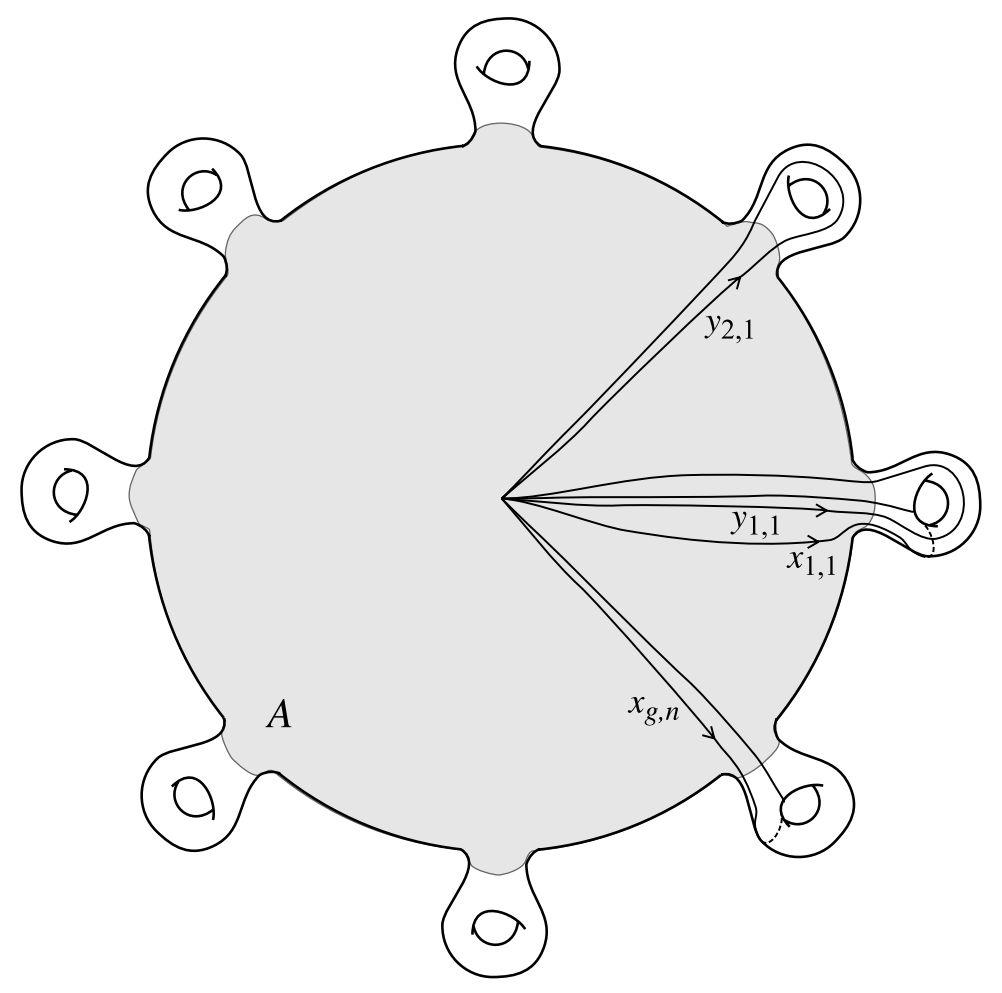

Figure 1. Standard generators of a surface of genus $g n$.

The $r+g$ relations give us $r+g$ immersed curves $\left\{\gamma_{i}\right\}$ on the surface $F$. We identify $F$ with a fiber of $N$ to view $\gamma_{i} \subseteq N$, and then the product of these curves with $S^{1}$ give us $r+g$ tori $T_{i}:=\gamma_{i} \times S^{1} \subseteq N \times S^{1}$. Figure 1 below is taken from [BK2], and here we see examples of how occurrences of $x_{i}$ and $y_{j}$ give us curves on this surface. Concatenating these words as specified by the relations determines the curves $\gamma_{i}$.

We supress the details, as they are given explicitly in [BK2], but we mention that the positivity of the powers of the generators in the relations ensures that we can perturb these tori, so that they are symplectic. In order to make the construction work, the tori $T_{i}$ need to be disjoint, and these curves need to be mapped into different parts of the surface. In [BK2], a second subscript is used, as is seen in Figure 1, to keep track of this data, but only the first subscript will determine to which element a loop corresponds in the fundamental group, as we demonstrate below. In this notation, a presentation for $\pi_{1}(F)$ is given by

$$
\pi_{1}(F)=\left\langle\begin{array}{c}
x_{1,1}, y_{1,1}, \ldots, x_{g, 1}, y_{g, 1}, \\
x_{1,2}, y_{1,2}, \ldots, x_{g, 2}, y_{g, 2}, \ldots, \\
x_{1, n}, y_{1, n}, \ldots, x_{g, n}, y_{g, n}
\end{array}\right| \prod_{l=1}^{n} g
$$


There is one more torus $T_{0}$ which we consider, defined as the product of the circle $\tau$ and the circle $\{*\} \times S^{1}$ corresponding to $s$.

The presentation for $\pi_{1}\left(N \times S^{1}\right)$ is

$$
\left.\left\langle\pi_{1} F, t\right| R_{*}^{g}(x)=t x t^{-1} \text { for } x \in \pi_{1} F\right\rangle \times\langle s\rangle .
$$

The idea underlying the construction is to take the symplectic sum of $N \times S^{1}$ along the tori $T_{0}, \ldots, T_{r+g}$ with other symplectic manifolds to kill the homotopy classes of loops that generate $\pi_{1}\left(T_{i}\right)$ while not introducing additional generators to the fundamental group of our manifold.

Recall that the symplectic sum $[\mathbf{G}]$ involves removing the tubular neighborhoods of two symplectic surfaces in two symplectic 4-manifolds and identifying their boundaries in a fiber-preserving manner, covering a diffeomorphism of the surfaces. The resulting 4-manifold admits a symplectic form, agreeing with the symplectic forms of the original two symplectic 4-manifolds away from the surfaces where the symplectic sum was performed. In order to perform this operation, the symplectic surfaces in each manifold must have normal bundles of oppositely signed Euler class, but this condition is satisfied in this case, since our tori in each manifold have trivial normal bundles.

Note that symplectic summing in such a manner along $T_{0}$ to kill $s$ and $t$ gives us a presentation

$$
\left.\left\langle\pi_{1} F\right| R_{*}^{g}(x)=x \text { for } x \in \pi_{1} F\right\rangle .
$$

This is actually a consequence of Lemma 4.1. The surface $F$ has genus $n g$, and so $\pi_{1}(F)$ has $2 n g$ generators. After quotienting by the relation $R_{*}^{g}(x)=x, 2 g$ generators remain, which we naturally identify with $x_{1}, \ldots, x_{g}, y_{1}, \ldots, y_{g}$. Note that once we sum along the tori $T_{r+1}, \ldots, T_{r+g}$, the relations $x_{i} y_{i}=1$ imply the surface relation of $\pi_{1}(F)$, since the surface relation is a product of commutators $\left[x_{i}, y_{i}\right]$. Hence, once we sum along the remaining tori, $T_{1}, \ldots, T_{g}$, we have a symplectic 4 -manifold with fundamental group generated by $x_{1}, \ldots, x_{g}, y_{1}, \ldots, y_{g}$ and relations $\tilde{w}_{1}, \ldots, \tilde{w}_{r}$, so that this group is $G$. We elaborate on these latter details in Section 4.

\section{Symplectic manifolds that kill $\pi_{1}$}

The construction utilizes two previous constructions of symplectic manifolds. The first is a symplectic manifold homeomorphic to $3 \mathbb{C P}^{2} \# 5 \overline{\mathbb{C P}}^{2}$ constructed in [BK1], which we will denote by $Y$. The useful thing about this manifold is that it contains two essential symplectic tori with trivial normal bundles and simply connected complement. This is the statement of Theorem 18 of [BK1]. The others are symplectic manifolds constructed in [FPS] which are homology $S^{2} \times S^{2}$ 's. The utilization of the former manifold will be straightforward, but we need to discuss the latter in a little detail. 


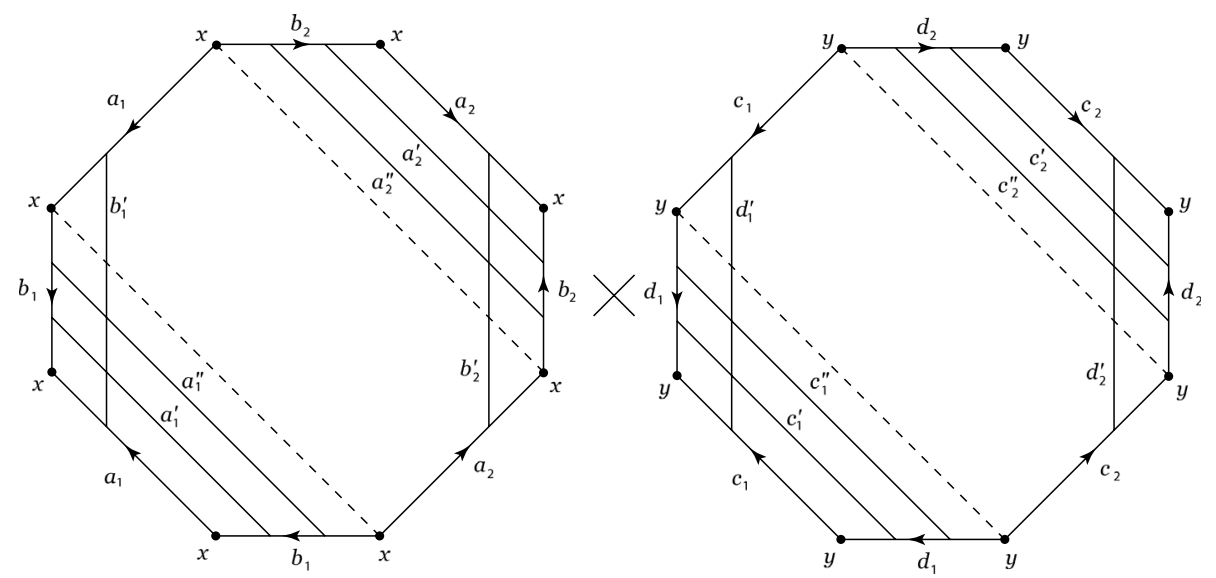

Figure 2. Tori in $\Sigma_{2} \times \Sigma_{2}$ which yield a homology $S^{2} \times S^{2}$ when surgered.

As described in [FPS], we will consider a product $\Sigma_{2} \times \Sigma_{2}$ of genus 2 surfaces, and a configuration of eight disjoint Lagrangian tori. We let $a_{1}, a_{2}, b_{1}, b_{2}, c_{1}, c_{2}, d_{1}, d_{2}$ denote generators of $\pi_{1}\left(\Sigma_{2} \times \Sigma_{2}\right)$, but we do not want the basepoint used to specify the fundamental group to be $(x, y)$, the usual basepoint. We address this issue later in Section 3. See Figure 2, which is taken from [FPS].

By performing Luttinger surgery $([\mathbf{A D K}],[\mathbf{L u t}])$ on seven of these tori, we obtain a symplectic manifold, which we call $V$, with a pair of dual Lagrangian tori and fundamental group generated by the eight elements mentioned, and having the relations

$$
\begin{aligned}
& a_{1}=\left[b_{1}^{-1}, d_{1}^{-1}\right] \quad b_{1}=\left[a_{1}^{-1}, d_{1}\right] \quad c_{1}=\left[d_{1}^{-1}, b_{2}^{-1}\right] \quad d_{1}=\left[c_{1}^{-1}, b_{2}\right] \\
& b_{2}=\left[a_{2}^{-1}, d_{2}\right] \quad c_{2}=\left[d_{2}^{-1}, b_{1}^{-1}\right] \quad d_{2}=\left[c_{2}^{-1}, b_{1}\right]
\end{aligned}
$$

in addition to some other relations.

The relations (3.1) result from performing surgeries on tori, each surgery being a "parametrized Dehn surgery." Each one of these surgeries can be described by specifying a triple consisting of the torus surgered, the curve along which the surgery is performed, and a rational number describing which surgery to perform, in the same way that a rational number specifies a Dehn surgery. Specifically, the relations of (3.1) result from performing the seven respective surgeries

$$
\begin{array}{rrrr}
\left(a_{1}^{\prime} \times c_{1}^{\prime}, a_{1}^{\prime},-1\right) & \left(b_{1}^{\prime} \times c_{1}^{\prime \prime}, b_{1}^{\prime},-1\right) & \left(a_{2}^{\prime} \times c_{1}^{\prime}, c_{1}^{\prime},+1\right) & \left(a_{2}^{\prime \prime} \times d_{1}^{\prime}, d_{1}^{\prime},+1\right) \\
& \left(b_{2}^{\prime} \times c_{2}^{\prime \prime}, b_{2}^{\prime},-1\right) & \left(a_{1}^{\prime} \times c_{2}^{\prime}, c_{2}^{\prime},+1\right) & \left(a_{1}^{\prime \prime} \times d_{2}^{\prime}, d_{2}^{\prime},+1\right) .
\end{array}
$$

The seven relations of (3.1) and corresponding surgeries are taken verbatim from [FPS], resulting from performing seven of the eight torus surgeries 
as done in $[\mathbf{F P S}]$. Note that if we introduce the relation $a_{2}=1$, then we will obtain a presentation of the trivial group. We will use this observation shortly.

Now let $V^{\prime}$ denote the complement of one of the two essential Lagrangian tori (call it $L$ ) in $V$. Specifically, let $L$ be the torus $a_{2}^{\prime} \times c_{2}^{\prime}$ in Figure 2. Note that this is the torus used to kill $a_{2}$ in homology in [FPS]. Then $\pi_{1}\left(V^{\prime}\right)$ is generated by the eight elements $a_{1}, \ldots, d_{2}$ and several elements $\epsilon_{1}, \ldots, \epsilon_{k}$, each of which is conjugate to $\mu$, a fixed, based meridian of the removed Lagrangian torus $L$. To be careful, we consider these elements in $\pi_{1}\left(V^{\prime}\right)$ to be based at a point $v \in \partial(\nu L)$.

Let $\beta$ be a path in $V^{\prime}$ from $(x, y)$ to $v \in \partial(\nu L)$ contained in the $b_{2} \times d_{2}$ torus, which intersects $L$ transversely in one point. Then conjugating the standard paths representing generators of $\pi_{1}\left(\Sigma_{2} \times \Sigma_{2},(x, y)\right)$ by $\beta$, we obtain paths based at $v$. In Section 4 , the symbols $a_{1}, a_{2}, b_{1}, b_{2}, c_{1}, c_{2}, d_{1}, d_{2}$ refer to elements of $\pi_{1}\left(V^{\prime}, v\right)$. Note that with this new basepoint $v$, the relations of (3.1) still hold in $V^{\prime}$.

\section{Putting the pieces together}

In Section 2, we identified symplectic tori $T_{0}, \ldots, T_{r+g}$ in $N \times S^{1}$. Recall that $Y$ contains a symplectic torus of algebraic square 0 , with simply connected complement. We symplectic sum $Y$ with $N \times S^{1}$ along $T_{0}$, giving us a symplectic manifold $W$.

\section{Lemma 4.1.}

$$
\pi_{1}(W)=\left\langle\pi_{1}\left(N \times S^{1}\right) \mid s, t\right\rangle .
$$

Proof. Note that here it is important that the complement of the symplectic torus $T$ in $Y$ is simply connected. To be precise, we apply the Seifert-van Kampen Theorem to compute $\pi_{1}(W)$. $[\mathbf{H}]$ The two manifolds that we use are $N \times S^{1} \backslash \nu T_{0}$ and $Y \backslash \nu T$, where $\nu T_{0}$ and $\nu T$ denote tubular neighborhoods of $T_{0}$ and $T$, respectively, and the intersection of these manifolds is an open neighborhood of $T^{3}$. Applying the Seifert-van Kampen Theorem,

$$
\pi_{1}(W)=\frac{\pi_{1}\left(N \times S^{1} \backslash \nu T_{0}\right) * \pi_{1}(Y \backslash \nu T)}{\left\langle\left\langle i_{1}(\alpha) i_{2}(\alpha)^{-1}: \alpha \in \pi_{1}\left(T^{3}\right)\right\rangle\right\rangle},
$$

where $T^{3}$ denotes the identified 3 -torus boundaries of $N \times S^{1} \backslash \nu T_{0}$ and $Y \backslash \nu T$, and $i_{1}: \pi_{1}\left(T^{3}\right) \rightarrow \pi_{1}\left(N \times S^{1} \backslash \nu T_{0}\right)$ and $i_{2}: \pi_{1}\left(T^{3}\right) \rightarrow \pi_{1}(Y \backslash \nu T)$ denote the maps on $\pi_{1}$ induced by the inclusions $T^{3} \hookrightarrow N \times S^{1} \backslash \nu T_{0}$ and $T^{3} \hookrightarrow Y \backslash \nu T$, respectively. The double angled brackets indicate the normal subgroup generated by those elements.

Since $\pi_{1}(Y \backslash \nu T)=1$, we have

$$
\pi_{1}(W)=\frac{\pi_{1}\left(N \times S^{1} \backslash \nu T_{0}\right)}{\left\langle\left\langle i_{1}(\alpha): \alpha \in \pi_{1}\left(T^{3}\right)\right\rangle\right\rangle} .
$$


Let the generators of $\pi_{1}\left(T^{3}\right)$ be denoted by $s_{T}, t_{T}$, and $\mu_{T}$, where $s_{T}$ and $t_{T}$ are homotopic to $s$ and $t$, respectively, in $N \times S^{1} \backslash \nu T_{0}$. In $\pi_{1}\left(N \times S^{1} \backslash \nu T_{0}\right)$, there is a normal generator $\mu$ of the meridian of $T_{0}$, but notice that in $\pi_{1}(W)$, $\mu=i_{1}\left(\mu_{T}\right)=1$. Also, since $s=i_{1}\left(s_{T}\right)=1$ and $t=i_{1}\left(t_{T}\right)=1$, we have proven the lemma.

Recall that a presentation of $\pi_{1}\left(N \times S^{1}\right)$ is

$$
\left.\pi_{1}\left(N \times S^{1}\right)=\left\langle\pi_{1} F, t\right| R_{*}^{g}(x)=t x t^{-1} \text { for } x \in \pi_{1} F\right\rangle \times\langle s\rangle .
$$

so that after applying Lemma 4.1, killing the generators $s$ and $t$, we have

$$
\left.\pi_{1}(W)=\left\langle\pi_{1} F\right| R_{*}^{g}(x)=x \text { for } x \in \pi_{1} F\right\rangle .
$$

In the notation of $(2.2), R_{*}^{g}\left(x_{k, l}\right)=x_{k, l+1}$ and $R_{*}^{g}\left(y_{k, l}\right)=y_{k, l+1}$, where the addition on the second factor is taken modulo $n$ (with $n+1=1$ ). Incorporating the presentation of $\pi_{1}(F)$ from (2.2), we have this presentation of $\pi_{1}(W)$ :

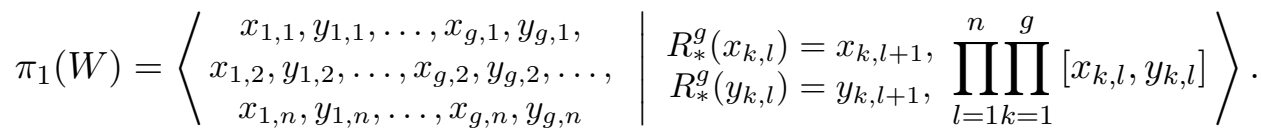

Hence, we assign the notation $x_{k}=x_{k, l}$ and $y_{k}=y_{k, l}$, as these elements are equal in $\pi_{1}(W)$ for different $l$, and we obtain the simplified presentation of the following lemma.

Lemma 4.2.

$$
\pi_{1}(W)=\left\langle x_{1}, y_{1}, x_{2}, y_{2}, \ldots, x_{g}, y_{g} \mid \prod_{l=1}^{n} \prod_{k=1}^{g}\left[x_{k}, y_{k}\right]\right\rangle .
$$

The next step is to perform the remaining $r+g$ symplectic sums to introduce the relations that will yield our presentation of $G$. We perturb the symplectic form on $V$, as described in $[\mathbf{G}]$, so that the torus $L \subseteq V$ is now symplectic. Now based curves in $V$ representing generators of $\pi_{1}(L)$ are homotopic to $a_{2}$ and $c_{2}$ in $\pi_{1}(V)$. We also see that we can choose $\mu$ to be $\left[b_{2}^{-1}, d_{2}^{-1}\right]$, as can be seen in Figure 2. Recall that $V^{\prime}$ denotes the complement of $L$ in $V$.

We perform symplectic sums of $W$ with $r+g$ copies of $V$ (each copy denoted by $V_{i}$ ), via the diffeomorphism that identifies the $\{*\} \times S^{1}$ curve in $T_{i}$ with $a_{2, i}$ in $V_{i}$, and the $\gamma_{i} \times\{*\}$ curve in $T_{i}$ with $c_{2, i}$ in $V_{i}$. Here $a_{2, i}$ and $c_{2, i}$ denote Lagrangian pushoffs of $a_{2}^{\prime}$ and $c_{2}^{\prime}$, respectively, contained in $\partial\left(\nu L_{i}\right)$ in $V_{i}$. Let $\phi_{i}$ denote this diffeomorphism between $\partial V^{\prime}$ and the $i$ th boundary component of

$$
W^{\prime}:=W \backslash \bigcup_{i=1}^{r+g} \nu T_{i}
$$


It is known that this construction can be performed symplectically $[\mathbf{G}]$, but we only need to consider it topologically to determine $\pi_{1}$.

Lemma 4.3. Let $X$ be the result of performing the symplectic sum of $W$ with the $r+g$ manifolds $V_{i}$. Then the fundamental group of $X$ is

$$
\pi_{1}(X)=\frac{\pi_{1}\left(W^{\prime}\right) * \pi_{1}\left(V_{1}^{\prime}\right) * \cdots * \pi_{1}\left(V_{r+g}^{\prime}\right)}{\left\langle\left\langle a_{2, i} \hat{s}_{i}^{-1}, c_{2, i} \hat{\gamma}_{i}^{-1}, \mu_{i} \hat{m}_{i}^{-1}\right\rangle\right\rangle} .
$$

In the statement of Lemma 4.3, $\left\langle\left\langle a_{2, i} \hat{s}_{i}^{-1}, c_{2, i} \hat{\gamma}_{i}^{-1}, \mu_{i} \hat{m}_{i}^{-1}\right\rangle\right\rangle$ is the normal subgroup of $\pi_{1}\left(W^{\prime}\right) * \pi_{1}\left(V_{1}^{\prime}\right) * \cdots * \pi_{1}\left(V_{r+g}^{\prime}\right)$ generated by, for each $i$, the elements $a_{2, i} \hat{s}_{i}^{-1}, c_{2, i} \hat{\gamma}_{i}^{-1}$, and $\mu_{i} \hat{m}_{i}^{-1}$, where $\mu_{i}$ denotes an element represented by a based meridian of $L_{i}$ in $V_{i}^{\prime}$. There is a subscript $i$ since we are performing $r+g$ symplectic sums with $V$. Here $\hat{\gamma}_{i}$ is represented by a based loop in $W^{\prime}$ which is (freely) homotopic to a curve $\gamma_{i} \times\{*\}$ in the boundary of a regular neighborhood of $T_{i}=\gamma_{i} \times S^{1}$. Also, $\hat{s}_{i}$ is represented by a based curve in $W^{\prime}$, which is (freely) homotopic to $\{*\} \times S^{1}$ on the boundary of a regular neighborhood of $T_{i}=\gamma_{i} \times S^{1}$. Finally, each $\hat{m}_{i}$ is a based meridian of the torus $T_{i}$ in $W^{\prime}$.

Proof of Lemma 4.3. Here we need to be careful with our application of the Seifert-van Kampen Theorem. The sets in question must be open and share a common basepoint. To ensure this, we choose a basepoint $w_{0} \in W^{\prime}$, and we have points $v_{i} \in \partial \nu L_{i} \subseteq \partial V_{i}^{\prime}$ corresponding to the basepoint $v$ mentioned in Section 3, and we fix paths $\eta_{i}$ from $w_{0}$ to $\phi\left(v_{i}\right)$. We set $\bar{V}_{i} \subseteq X$ equal to $V_{i}^{\prime}$ union a neighborhood of $\eta_{i}$. Hence $\bar{V}_{i}$ contains the basepoint $w_{0}$, and $\pi_{1}\left(\bar{V}_{i}, w_{0}\right) \cong \pi_{1}\left(V^{\prime}, v\right)$. Furthermore, we use the same notation for an element of $\pi_{1}\left(\bar{V}_{i}, w_{0}\right)$ as we do for a corresponding element of $\pi_{1}\left(V_{i}^{\prime}, v_{i}\right)$, under the usual correlation of conjugating by the path $\eta_{i}$.

Now we are in a position to apply the Seifert-van Kampen Theorem, using the open sets $W^{\prime}, \bar{V}_{1}, \ldots, \bar{V}_{r+g}$, which intersect pairwise in connected sets. The Seifert-van Kampen Theorem gives us that

$$
\pi_{1}(X)=\frac{\pi_{1}\left(W^{\prime}\right) * \pi_{1}\left(\bar{V}_{1}\right) * \cdots * \pi_{1}\left(\bar{V}_{r+g}\right)}{\left\langle\left\langle\iota_{W}(\gamma) \iota_{V i}(\gamma)^{-1} \mid \gamma \in \pi_{1}\left(W^{\prime} \cap \bar{V}_{i}\right)\right\rangle\right\rangle},
$$

where $\iota_{W}: \pi_{1}\left(W^{\prime} \cap \bar{V}_{i}\right) \rightarrow \pi_{1}\left(W^{\prime}\right)$ and $\iota_{V i}: \pi_{1}\left(W^{\prime} \cap \bar{V}_{i}\right) \rightarrow \pi_{1}\left(\bar{V}_{i}\right)$ are the maps on $\pi_{1}$ induced by the inclusions $W^{\prime} \cap \bar{V}_{i} \hookrightarrow W^{\prime}$ and $W^{\prime} \cap \bar{V}_{i} \hookrightarrow \bar{V}_{i}$, respectively. Note that these are the only elements that we will have in the quotient by applying the Seifert-van Kampen Theorem, because the other intersections between $\bar{V}_{i}$ and $\bar{V}_{j}$ for $i \neq j$ are contractible.

For a fixed $i$, let us consider the group

$$
\left\langle\left\langle\iota_{W}(\gamma) \iota_{V i}(\gamma)^{-1} \mid \gamma \in \pi_{1}\left(W^{\prime} \cap \bar{V}_{i}\right)\right\rangle\right\rangle
$$


As $W^{\prime} \cap \bar{V}_{i}$ is homotopy equivalent to a 3 -torus, $\pi_{1}\left(W^{\prime} \cap \bar{V}_{i}, w_{0}\right)$ is generated by three elements. Let us denote these three elements by $a, c$, and $\mu$, so that, using our earlier notation, $\iota_{V i}(a)=a_{2, i}, \iota_{V i}(c)=c_{2, i}$, and $\iota_{V i}(\mu)=\mu_{i}$. By the definition of $\phi_{i}$, this means that $\iota_{W}(a)=\hat{s}_{i}, \iota_{W}(c)=\hat{\gamma}_{i}$, and $\iota_{W}(\mu)=\hat{m}_{i}$. Therefore (4.4) is the same as

$$
\left\langle\left\langle a_{2, i} \hat{s}_{i}^{-1}, c_{2, i} \hat{\gamma}_{i}^{-1}, \mu_{i} \hat{m}_{i}^{-1}\right\rangle\right\rangle .
$$

This completes the proof of Lemma 4.3.

We will now show that $\hat{s}_{i}$ is homotopic to $s$. This follows since both $\hat{s}_{i}$ and $s$ are curves in $W^{\prime}$ of the form $\{*\} \times S^{1}$, and we can certainly find a path between the points in the first factor. To be precise, let

$$
W^{\prime \prime}:=\left(N \times S^{1}\right) \backslash \bigcup_{i=0}^{r+g} \nu T_{i} .
$$

Each $T_{i} \subseteq N \times S^{1}$ is a torus of the form $\gamma \times S^{1}$ for some curve $\gamma \subseteq N$. So, finding a homotopy between $\hat{s}_{i}$ and $s$ in $W^{\prime \prime}$ reduces to finding a path between two points in $N$ minus a union of circles. This is certainly possible, as circles have codimension 2 in $N$. As $W^{\prime \prime} \subseteq W^{\prime}$, this gives us a homotopy in $W^{\prime}$ as well, but in $W^{\prime}, s$ is nullhomotopic. The argument used here only shows that these curves are freely homotopic, but that is sufficient for our purposes since $s$ is nullhomotopic and so $\hat{s}_{i}$, being freely homotopic to $s$, is also based nullhomotopic.

We now continue to simplify our expression of $\pi_{1}(X)$.

\section{Lemma 4.4.}

$$
\pi_{1}(X)=\frac{\pi_{1}\left(W^{\prime}\right)}{\left\langle\left\langle\hat{\gamma}_{i}, \hat{m}_{i}\right\rangle\right\rangle} .
$$

Proof. Notice that $\pi_{1}\left(V_{i}^{\prime}\right)$ is generated by $a_{1, i}, \ldots, d_{2, i}, \epsilon_{1, i}, \ldots, \epsilon_{k_{i}, i}$, where each $\epsilon_{l, i}$ is conjugate to $\mu_{i}$. As mentioned in Section 3, the relations (3.1) hold in $\pi_{1}\left(V_{i}^{\prime}, v_{i}\right)$.

In $\pi_{1}(X), a_{2, i}=\hat{s}_{i}=1$ ( since $\hat{s}_{i}$ is nullhomotopic). We can work our way through the relations (3.1) to show that the elements $a_{1, i}, \ldots, d_{2, i}$ are trivial in $\pi_{1}(X)$. Starting with the fact that $a_{2, i}=1$, we have $b_{2, i}=\left[a_{2, i}^{-1}, d_{2, i}\right]=$ $\left[1, d_{2, i}\right]=1$. Next, $c_{1, i}=\left[d_{1, i}^{-1}, b_{2, i}^{-1}\right]=\left[d_{1, i}^{-1}, 1\right]=1$ and $d_{1, i}=\left[c_{1, i}^{-1}, b_{2, i}\right]=$ $\left[c_{1, i}^{-1}, 1\right]=1$. So far we have killed three of the generators using the relations of (3.1). Using that $d_{1, i}=1, b_{1, i}=\left[a_{1, i}^{-1}, d_{1, i}\right]=\left[a_{1, i}^{-1}, 1\right]=1$. It follows that $a_{1, i}=\left[b_{1, i}^{-1}, d_{1, i}^{-1}\right]=[1,1]=1$. It remains to show that $c_{2, i}$ and $d_{2, i}$ are trivial, which follows since $c_{2, i}=\left[d_{2, i}^{-1}, b_{1, i}^{-1}\right]=\left[d_{2, i}^{-1}, 1\right]=1$, and $d_{2, i}=\left[c_{2, i}^{-1}, b_{1, i}\right]=$ $[1,1]=1$. 
Now $\mu_{i}=\left[b_{2, i}^{-1}, d_{2, i}^{-1}\right]=1$, and so $\epsilon_{1, i}, \ldots, \epsilon_{k_{i}, i}$ are all 1 as well. Hence we have killed all of the generators of $\pi_{1}\left(V_{i}^{\prime}\right)$, and applying Lemma 4.3, noting in particular that $c_{2, i}=1$, we have shown that

$$
\pi_{1}(X)=\frac{\pi_{1}\left(W^{\prime}\right) * 1 * \cdots * 1}{\left\langle\left\langle c_{2, i} \hat{\gamma}_{i}^{-1}, \mu_{i} \hat{m}_{i}^{-1}\right\rangle\right\rangle}=\frac{\pi_{1}\left(W^{\prime}\right)}{\left\langle\left\langle\hat{\gamma}_{i}, \hat{m}_{i}\right\rangle\right\rangle} .
$$

After each of these steps, we are finally in a position to show that the construction yields the desired fundamental group.

Theorem 4.5. $\pi_{1}(X)=G$.

Proof. Let $H$ be a set of curves in $W$ based at $w_{0}$ which miss the tori $T_{i}$, such that $H$ generates $\pi_{1}(W)$. We can consider $H$ to be a set of elements in $\pi_{1}\left(W^{\prime}\right)$ as well. Observe that $\pi_{1}\left(W^{\prime}\right)$ is generated by $H \cup\left\{\delta_{1}, \ldots, \delta_{k}\right\}$, where each $\delta_{i}$ is conjugate to one of the fixed set of meridians $\hat{m}_{1}, \ldots, \hat{m}_{r+g}$, where we recall $\hat{m}_{i}$ is a based meridian of $T_{i}$. From the fact that each $\hat{m}_{i}=1$, we have that $\left\{\delta_{1}, \ldots, \delta_{k}\right\}=\{1\}$.

Note that a representative curve $\hat{\gamma}_{i}$ is homotopic to $\gamma_{i}$ in $W$, but some of these homotopies may intersect the tori $T_{i}$. Hence, what we have is that $\hat{\gamma}_{i}$ and a conjugate of $\gamma_{i}$ differ by a product of conjugates of $\hat{m}_{l}$. As each of these $\hat{m}_{l}=1$, we have that $\hat{\gamma}_{i}$ is conjugate to $\gamma_{i}$ in $\pi_{1}(X)$.

So, now we have from Lemma 4.4 that

$$
\pi_{1}(X)=\pi_{1}(W) /\left\langle\left\langle\gamma_{i}\right\rangle\right\rangle,
$$

and as we have explained in Section 2, the $\gamma_{i}$ were chosen so that this group is $G$.

To make this precise, we work from the presentation of Lemma 4.2. Combining Lemma 4.2 with (4.5), and expanding $\gamma_{i}$ in our notation,

$$
\pi_{1}(X)=\frac{\left\langle x_{1}, y_{1}, x_{2}, y_{2}, \ldots, x_{g}, y_{g} \mid \prod_{l=1}^{n} \prod_{k=1}^{g}\left[x_{k}, y_{k}\right]\right\rangle}{\left\langle\left\langle\tilde{w}_{1}, \ldots, \tilde{w}_{r}, x_{1} y_{1}, \ldots, x_{g} y_{g}\right\rangle\right\rangle} .
$$

As explained at the end of Section 2, the relations $\left\{x_{i} y_{i}\right\}$ imply the surface relation $\prod_{l=1}^{n} \prod_{k=1}^{g}\left[x_{k}, y_{k}\right]$, and furthermore, the relations $\left\{x_{i} y_{i}\right\}$ can be used both to reduce $\tilde{w}_{i}$ back to $w_{i}$ and to eliminate $g$ of the generators $y_{1}, \ldots, y_{g}$. Hence we have from (4.6) that

$$
\pi_{1}(X)=\frac{\left\langle x_{1}, x_{2}, \ldots, x_{g}\right\rangle}{\left\langle\left\langle w_{1}, \ldots, w_{r}\right\rangle\right\rangle} .
$$


Now we see that the presentation of $\pi_{1}(X)$ in $(4.7)$ is the same as the presentation of $G$ in (2.1).

It remains to compute $\chi(X)$ and $\sigma(X)$ to complete the proof of Theorem 1.1. The Euler characteristic and signature add under symplectic sums along tori. Additionally, Luttinger surgeries preserve Euler characteristic and signature, and so we compute that

$$
\begin{aligned}
& \chi(V)=\chi\left(\Sigma_{2} \times \Sigma_{2}\right)=4 \\
& \sigma(V)=\sigma\left(\Sigma_{2} \times \Sigma_{2}\right)=0 .
\end{aligned}
$$

Now we use the additivity of Euler characteristic and signature to compute:

$$
\begin{aligned}
\chi(X) & =\chi\left(N \times S^{1}\right)+\chi(Y)+\sum_{i=1}^{r+g} \chi(V) \\
& =0+10+(r+g) \times 4,
\end{aligned}
$$

and

$$
\begin{aligned}
\sigma(X) & =\sigma\left(N \times S^{1}\right)+\sigma(Y)+\sum_{i=1}^{r+g} \sigma(V) \\
& =0+(-2)+(r+g) \times 0 .
\end{aligned}
$$

\section{References}

[ADK] D. Auroux, S. Donaldson and L. Katzarkov, Luttinger surgery along Langrangian tori and non-isotopy for singular symplectic plane curves, Math. Ann. 326 (2003), 185-203.

[BK1] S. Baldridge and P. Kirk, Constructions of small symplectic 4-manifolds using Luttinger surgery, J. Dif. Geom. 82(2) (2009), 317-361.

[BK2] S. Baldridge and P. Kirk, On symplectic 4-manifolds with prescribed fundamental group, Commentarii Mathematici Helvetici 82(4) (2007), 845-875.

[FPS] R. Fintushel, B. Doug Park and R. J. Stern, Reverse engineering small 4-manifolds, Algebraic and Geometric Topology 7 (2007), 2103-2116.

[FS] R. Fintushel and R. J. Stern, Six Lectures on Four 4-manifolds, 2006, Park City Mathematics Institute Graduate Summer School on Low Dimensional Topology.

[G] R. E. Gompf, New construction of symplectic manifolds, Ann. Math. 142(3) (1995), 527-595.

[H] A. Hatcher, Algebraic Topology, Cambridge University Press, 2001.

[Lut] K. M. Luttinger, Lagrangian tori in $\mathbb{R}^{4}$, J. Diff. Geom. 42 (1995), 220-228.

[MMS] J. Morgan, T. Mrowka and Z. Szabó, Product formulas along $T^{3}$ for Seiberg-Witten invariants, Math. Res. Lett. 4(6) (1997), 915-929.

[Th] W. P. Thurston, Some simple examples of symplectic manifolds. Proc. Amer. Math. Soc. 55(2) (1976), 467-468. 
Deptartment of Mathematics and Statistics, McMaster University, 1280 Main Street West, Hamilton, ON L8S 4K1, Canada

E-mail address: yazinski@math.mcmaster.ca

Received 05/05/2010, accepted 04/2/2011

The author would like to thank the referee for kindly making the observation mentioned in Remark 1.2. 